\title{
Mitochondrial/Nuclear Superstructures Drive Morphological Changes in Endometrial Epithelia by Pressure Exerted when Gas Vacuoles Form and Coalesce within Superstructures
}

\author{
Honoree Fleming \\ Castleton State College, Castleton, VT, USA \\ Email: honoree.fleming@castleton.edu
}

How to cite this paper: Fleming, H. (2018) Mitochondrial/Nuclear Superstructures Drive Morphological Changes in Endometrial Epithelia by Pressure Exerted when Gas Vacuoles Form and Coalesce within Superstructures. Advances in Bioscience and Biotechnology, 9, 224-242.

https://doi.org/10.4236/abb.2018.95016

Received: February 26, 2018

Accepted: May 28, 2018

Published: May 31, 2018

Copyright $\odot 2018$ by author and Scientific Research Publishing Inc. This work is licensed under the Creative Commons Attribution International License (CC BY 4.0).

http://creativecommons.org/licenses/by/4.0/

\section{(c) (i) Open Access}

\begin{abstract}
Mitochondria, usually ovoid structures no larger than 0.5 microns can fuse into structures that are 5 microns and larger such as nebenkern, spheroidal or cup-shaped mitochondria, and even mega-mitochondria. In studying differentiation of human endometrial epithelial cells, it became clear that formation of mitochondrial superstructures was an essential part of the process. In this paper, the origins, function, and demise of these superstructures called mitonucleons are described. In the course of reading papers about mitochondrial superstructures, it became obvious that there are important similarities, particularly with regard to function, between the mitonucleon and the nebenkern, a superstructure essential for dramatic tail elongation during spermatogenesis in grasshoppers, drosophila, and other insects. Close inspection of photomicrographs of differentiating mitonucleons during the first 12 hours suggests that gases build up in vacuoles within the mitochondrial superstructure creating pressure that elevates syncytial membranes and compresses nuclear aggregates contained within the mitonucleon.
\end{abstract}

\section{Keywords}

Mitochondrial, Nuclear Superstructures

\section{Introduction}

For almost as long as there have been microscopes (certainly more than a century), mitochondrial superstructures have been a topic of interest. Cell biologists started reporting on mitochondrial superstructures that they called nebenkern in 
grasshopper sperm as early as 1883 according to a 1922 review paper [1]. Culling from those papers and his own research, Bowen described the nebenkern as taking on "complicated and fantastic patterns" resulting in it being likened to "a blackberry, an onion, a ball of twine, and a skein of yarn" as changes in these mitochondrial superstructures accompany the process whereby round spermatids with a 12-micron-diameter develop into cylinders of the same diameter but elongated more than a hundred fold to almost $2 \mathrm{~mm}$ [2]. The pair of mitochondrial superstructures that similarly elongate has been thought for more than 100 years to be organizing centers for this amazing phenomenon of cell morphogenesis. As described in a recent review [2], the axoneme of the developing sperm is surrounded by ER membranes that associate intimately with the two giant mitochondrial derivatives of the nebenkern as they unfurl and elongate. Long tails must be beneficial for selection since mitochondrial superstructures predictably accompany spermatogenesis in many different insects [3].

Mitochondrial superstructures also accompany differentiation in the Ishikawa endometrial epithelial cell line [4]. The superstructures which we have called mitonucleons form in the initial hours following the addition of a differentiation stimulus [5] [6] to confluent, quiescent Ishikawa monolayers. In the ensuing 24 to 30 hours, changes brought about by mitochondrial superstructures result in fluid-enclosing hemispheres as shown in Figure 1(a). The structures stain for endogenous biotin linked to mitochondrial carboxylase enzymes [5]. Under the right conditions, and over a period of weeks, cells in the dome can grow out into gland-like structures as shown in Figure 1(b). While not as visually dramatic as spermatid tail elongation, these structural changes result in epithelial cells able to proliferate in an anchorage-independent manner and to form three dimensional structures that look like glands pushing up into the medium (Figure 1(b)) as they might push out into stroma in vivo. It is reasonable to assume that such changes are involved in the formation of glands every 28 days during the menstrual cycle. Furthermore, during this differentiation, the dome cells become

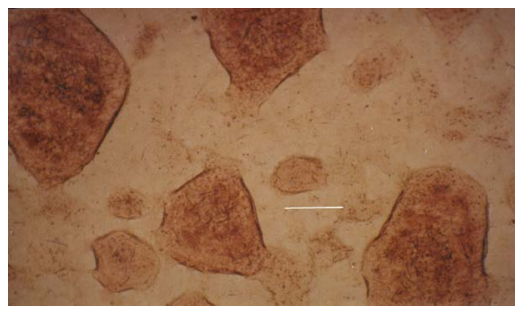

(a)

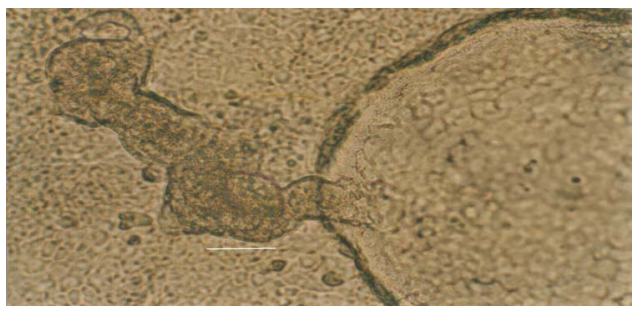

(b)

Figure 1. (a) Cellular hemispheres enclosing fluid begin to appear within 24 hours after stimulating differentiation in cultured endometrial epithelial cells. Domes continue to increase in size over time. The domes showed here are five days old. The endogenous biotin in domes has been shown [5] to be linked to mitochondrial carboxylases. (Bar $=100 \mathrm{mi}$ crons). (b) Under appropriate conditions, anchorage-free hemisphere cells continue to proliferate into gland-like structures as shown in this picture. A small outgrowth of cells on the dome elongated into this structure by day 28 following stimulation to differentiate. (Bar $=50$ microns). 
capable of synthesizing and secreting proteins that are not detected in the monolayer [7].

The first half of the sequence of events accompanying Ishikawa differentiation will be discussed in this paper. The earliest detectable change is the fusion of cells in regions of the monolayer throughout the dish, followed by aggregation of the resulting syncytial nuclei, and a dramatic increase in structures staining for the endogenous biotin of mitochondrial enzymes. Mitochondria, spherical and tubular, coalesce around each of the nuclear aggregates. When such structures (called giant mitochondria) were identified in human endometrial tissue, where they transiently appear around the time of ovulation, it was also noted that a single layer of endoplasmic reticulum surrounds these structures [8].

The physical evidence indicates that endometrial mitochondrial superstructures develop gas pressure capable of unfurling the apical membrane of the syncytium, perhaps in the same way that nebenkern unfurls membranes essential for the elongation of sperm tails. Furthermore, this may be the force responsible for the phenomenon of pycnotic nuclei.

\section{Results}

Fluid-filled hemispheres called domes resulting from Ishikawa epithelial differentiation are shown in Figure 1(a). Dramatic Increases in the mitochondrial carboxylases are evident in elevated endogenous biotin levels [5] [6]. Under the right conditions, dome cells will continue to proliferate in an anchorage-independent manner into gland-like structures as shown in Figure 1(b).

The earliest detectable changes in an Ishikawa monolayer undergoing differentiation are shown in Figure 2. Fifty or more cells in regions throughout the dish fuse to form syncytia within a couple of hours of stimulation. The structures in this differentiating monolayer were fixed and stained with hematoxylin and eosin and probed for endogenous biotin using peroxidase-linked streptavidin. Sharply delimited nuclei within, but at the edge of the syncytium, stain as darkly as surrounding monolayer nuclei. The nuclei that have moved toward the center of the structure stain more faintly suggesting structural changes that perhaps enable nuclear fusion. There are only faint traces of endogenous biotin in the syncytium one hour after it has been stimulated (Figure 2(a)).

The photomicrograph in Figure 2(b), taken 2 hours later in the process of dome formation, demonstrates an increase in detectable endogenous biotin (red arrows) in structures whose sizes range from 2 to 5 microns. The structures are interconnected to some extent and located close to nuclear aggregates.

An opportune observation of a syncytium after another 2 hours allowed detection of an early interaction of mitochondria with a nuclear aggregate (Figure 3). A transparent membrane staining for endogenous biotin has been elaborated around one of the multiple fusing nuclear aggregates [5], probably generated from an adjoining giant mitochondria. Such a structure has not previously been described although multiple reports of membranes generated from mitochondria 


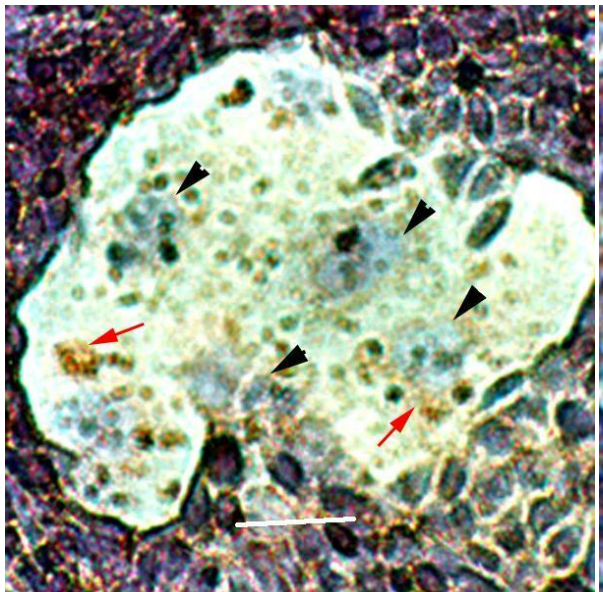

(a)

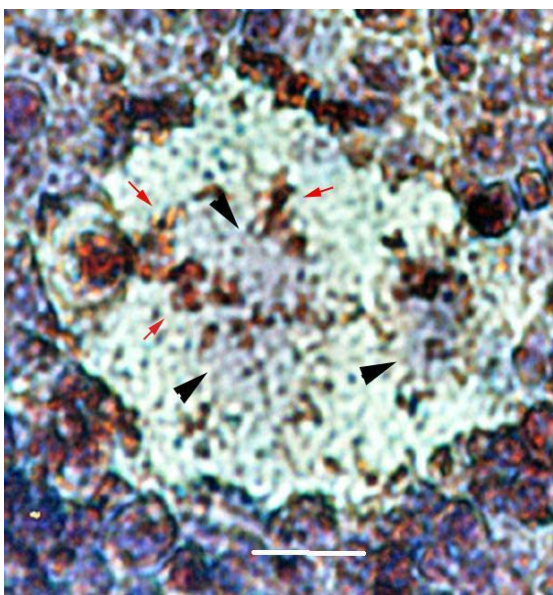

(b)

Figure 2. (a) The earliest changes in a monolayer stimulated to differentiate is cell fusion and syncytia formation, seen as early as within 3 hours. As mononucleated monolayer cells merge, the resulting nuclei fade and aggregate. Nuclei at one o'clock on the edge of the syncytium still have sharp boundaries that are not seen in the nuclei in the center (black arrowheads). The syncytium, also treated with streptavidin-linked peroxidase and AEC contain few giant mitochondria (red arrows). (Bar = 25 microns). (b) Within several more hours, endogenous biotin staining increases. Red arrows point to (some of the) particulate structures staining for endogenous biotin and contiguous with aggregated nuclei (black arrowheads). Meanwhile the nuclei appear to be fusing. (Bar $=25$ microns).

have appeared in the last decade including vesicles that form in HeLa cells and appear to participate in communication within the cell [9] [10] [11], as well as mitochondrial outer membranes that form autophagosomes in starved rat kidney cells [12].

In Figure 4, it is possible to detect 4 to 5 nuclear aggregates surrounded by structures that stain for endogenous biotin. The nuclear aggregate at 12 o'clock is the furthest along in a process that results in a giant spheroidal structure enveloping a nuclear aggregate as the syncytium detaches and becomes elevated [13].

Figure 4 demonstrates interaction of mitochondrial superstructures with nuclear aggregates building on the envelopment of the aggregate with a single mitochondrial membrane shown in Figure 3. The envelopment will become complete as the syncytium lifts off the plate. We discovered that the stain for endogenous biotin obscured another interesting characteristic of the mitochondrial structure as shown in Figure 5. Staining a monolayer stimulated to differentiate at about the same time as Figure 4 with only hematoxylin and eosin (Figure 5) reveals different stages in extensive vacuolization surrounding the nuclear aggregates.

Given the positioning of biotin staining in Figure 4, it must be assumed that these vacuoles are forming in the mitochondrial superstructures that surround the chromatin center of the mitonucleons. Observations of vacuoles are frequent in the literature of mitochondrial superstructures. One of the most thorough morphological studies [14] of the nebenkern accompanying spermatogenesis in 


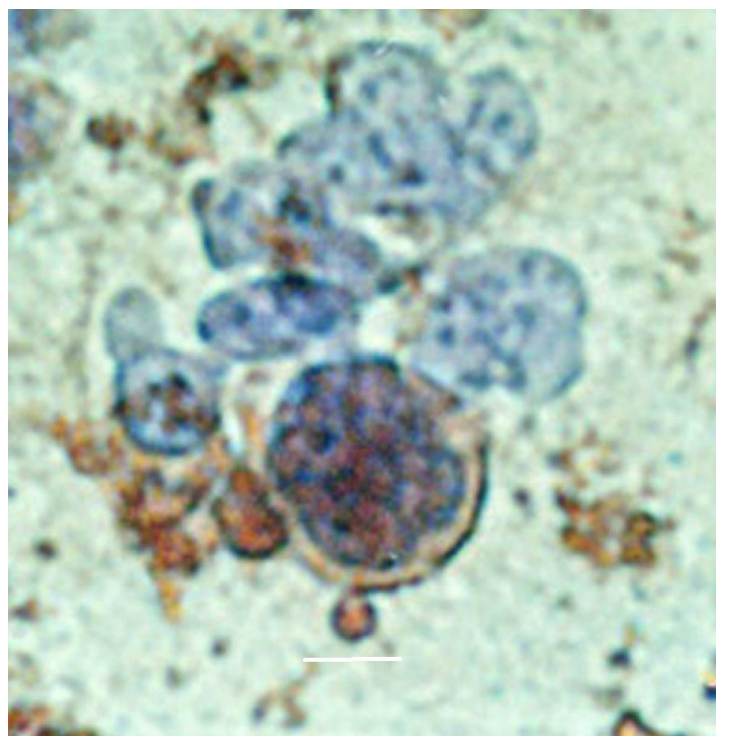

Figure 3. A membrane staining for biotin is elaborated around each nuclear aggregate. Structures staining for biotin outside the membrane at 6 o'clock and 8 o'clock are prime suspects for the origin of this transparent membrane. This structure is the first "step" in the creation of mitonucleons. Additional mitochondrial structures adhere to and eventually envelop the nuclear aggregate. (Bar $=25$ microns).

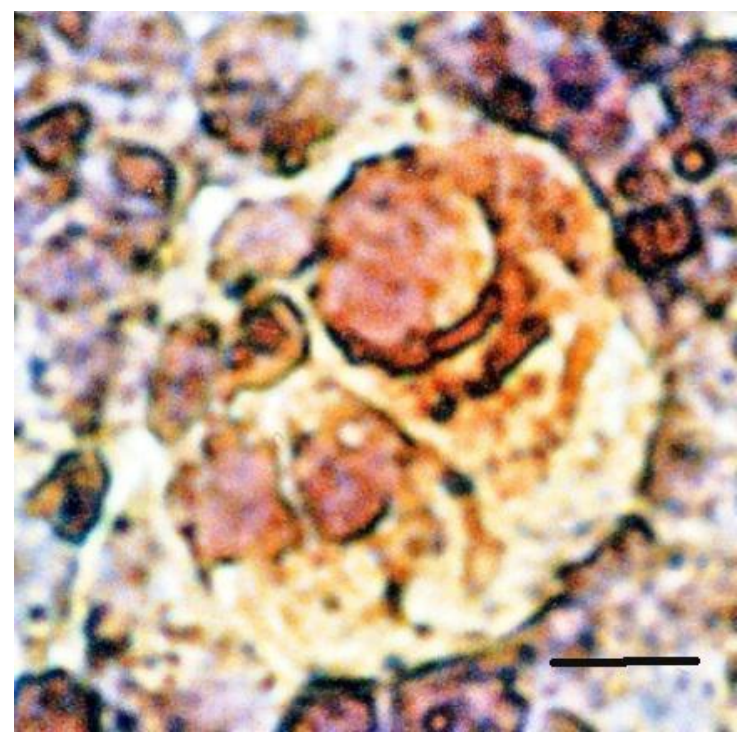

Figure 4. Structures staining for endogenous biotin contiguous with aggregated nuclei thicken as the process continues. The structure at 12 o'clock is a particularly significant C-shaped aggregate of material staining for endogenous biotin almost completely surrounding a nuclear aggregate. Two processes proceed from this point. The membranes resulting from fusing mitochondria will almost completely enclose the nuclear aggregate and it will compress the chromatin material to less than half its size in Figure 3. The details of spherical mitochondrial superstructures show an asymmetrical structure (hence cup shaped) with membranes thicker on one side of the nuclear aggregate and with an opening of the internal lumen out into the surrounding cytosol. (Bar $=25$ microns). 


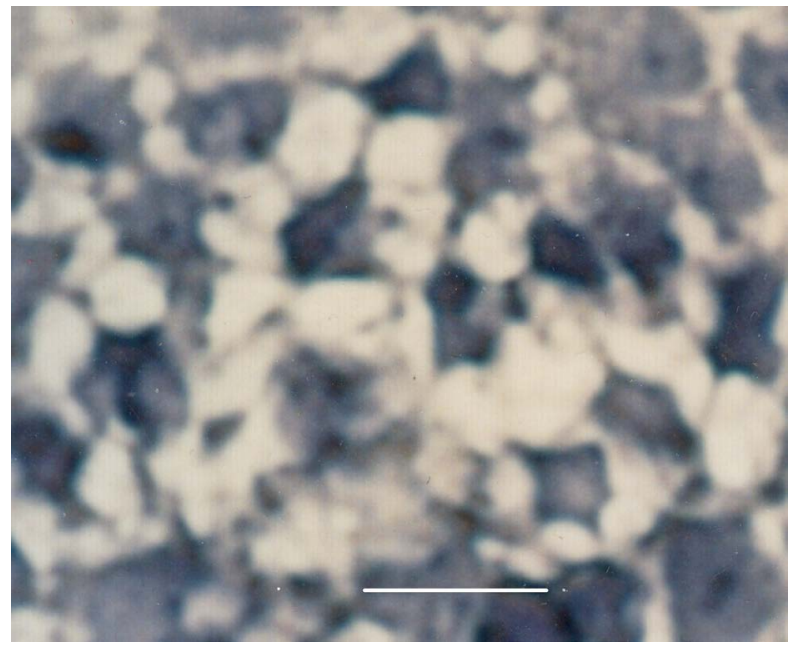

(a)

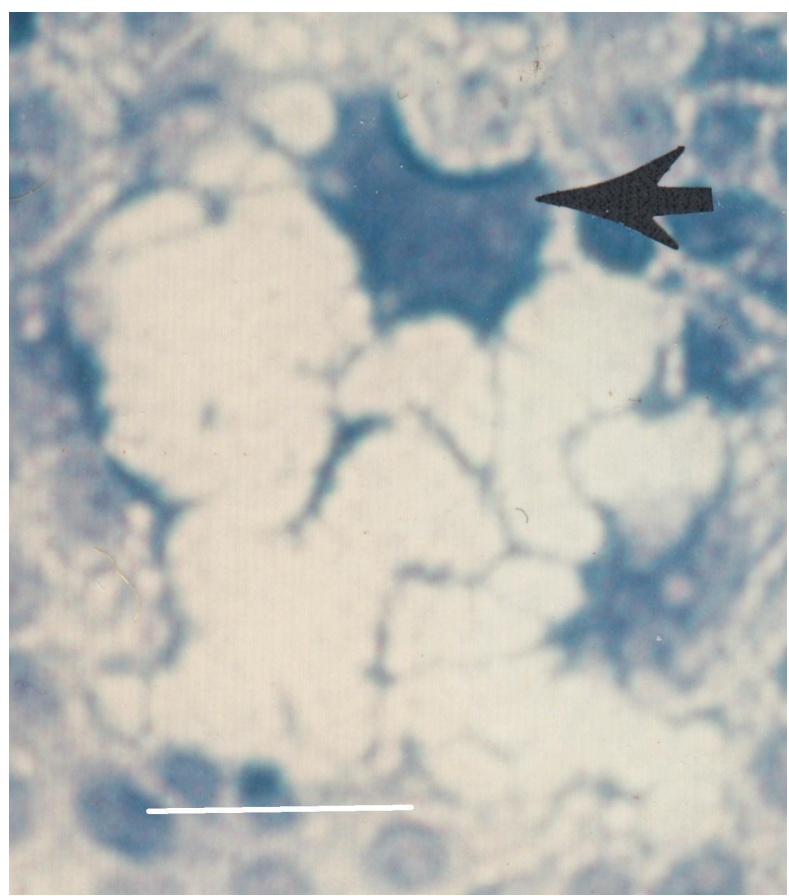

(b)

Figure 5. (a) Nuclear aggregates stained with hematoxylin appear to be surrounded by vacuoles. The stellate appearance of the nuclei suggests that these vacuoles exert pressure on the chromatin aggregates. (Bar $=25$ microns). (Bar $=25$ microns). (b) Larger vacuoles are evident later in the predome phase. The arrow points to a significant region of stained chromatin. Other regions staining for hematoxylin appear as thin lines between vacuoles. (Bar $=25$ microns).

the grasshopper shows vacuoles, similarly surrounding chromatin, that coalesce over time.

Vacuoles are larger in Figure 5(b) leading to the conclusion that the structures within the mitochondrial superstructure around the compressed chroma- 
tin material are subcompartments that can fuse to form larger vacuoles, and ultimately the large central vacuole characteristic of mitonucleons. Membranes among the enlarging gas vacuoles disappear while membranes between merging vacuoles and chromatin remain intact until the mitonucleon falls apart.

When a syncytium with an unfurling apical membrane (Figure 6) is stained for chromatin and for endogenous biotin, mitonucleons are observed in the center of the structure elevating with the membrane. These structures are surrounded by additional particulate biotin staining material outside of the mitonucleons suggesting that biogenesis of typical mitochondria occurs at the same time as the differentiation of the mitochondrial superstructures.

Since the upward thrust of the apical membrane appears to be a driver of the differentiation, we tried to capture an image suggestive of the extent of unfurling of apical membrane projections in living cultures. The attempt was made with a culture that was neither fixed nor stained (Figure 7). With the objective focused on the aggregated chromatin pressed against the unfurling apical membrane, the monolayer was clearly out of focus. Additionally small dark structures surrounding the chromatin were flattened against, and rising with, the unfurling membrane. These are the appropriate size to be centrioles and the phenomenon of mitonucleon formation may involve some kind of interaction between mitochondria, chromatin, and microtubules. Such a role for microtubules was suggested by Kedzior's study eliciting spheroidal mitochondrial superstructures surrounding

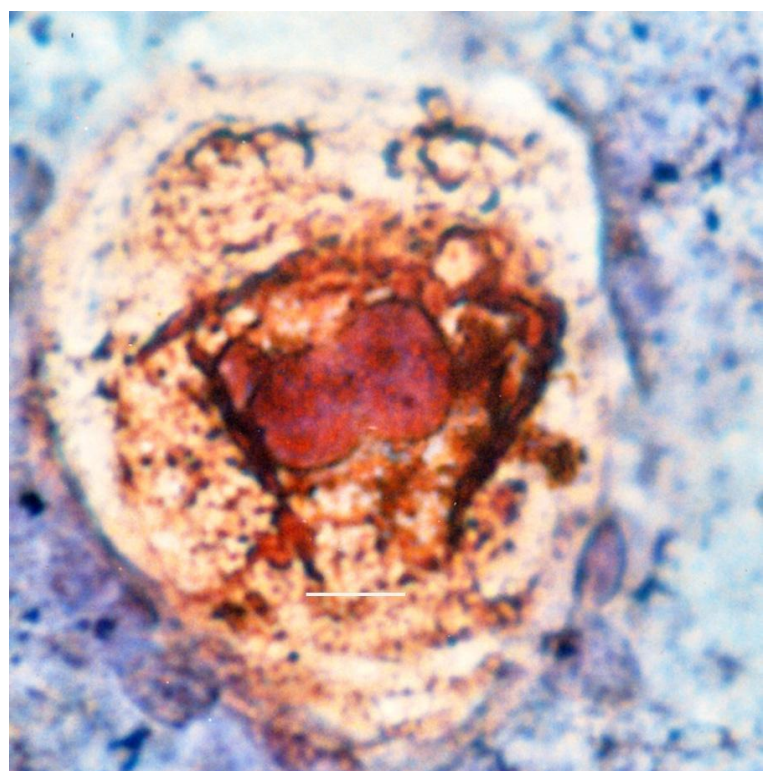

Figure 6. Multiple mitochondrial superstructures form within a syncytium, each surrounding a nuclear aggregate. Additionally the syncytium is filled with particulate structures that stain for biotin whose size is smaller than the approximately 25 micron mitonucleon, but larger than a typical 0.5 micron mitochondria. But the mitonucleons, ( 3 or 4 in this syncytium) will change dramatically in the next few hours. (Bar $=25$ microns). 


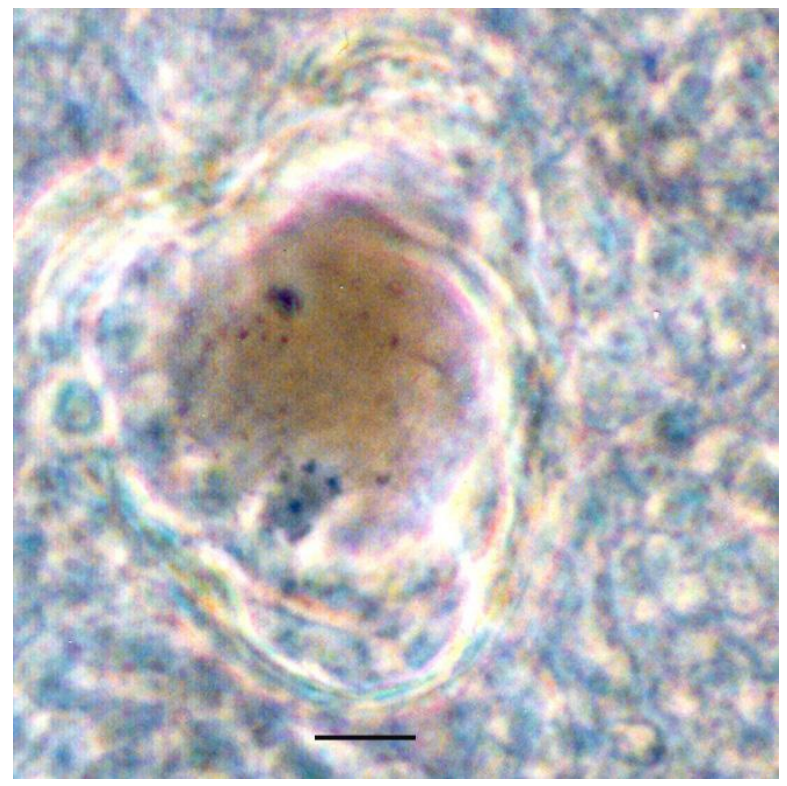

Figure 7. The microscope was focused on the nuclear structure flattened against a membranous projection rising out of syncytium. Monolayer cells are out of focus. If this structure were fixed, it would resemble the structures in Figure 8. Light microscopy as opposed to phase contrast microscopy was used, together with a blue filter, to obtain an image that is suggestive of a cone-shaped membranous projection. Bar $=25$ microns.

chromatin in cultured cells by adding microtubule active drugs to monolayers. [15].

Fixing structures at this predome stage (Figure 8) results in the loss of much of the apical membrane elevation but allows a better look at what is happening inside and outside of the mitonucleon projections. The aggregated chromatin material is flattened to one side and against the mitonucleon membrane, looking very much like the "signet-ring cells" that were first identified in cancer tissue and assumed to be pathological [16]. Within the envelope of that membrane dark structures that look like centrioles are observable. Our results raise the possibility that at least some of the time "signet-ring cells" are actually mitonucleons with large central gas vacuoles that compress the chromatin and flatten it against the mitonucleon membrane that is pushing up on the apical membrane.

The term pycnotic has been used to describe the appearance of nuclei such as those observed in Figure 8. It is assumed that chromatin or heterochromatin becomes pycnotic due to pressure, the source of which has not been identified before now. Our results suggest that pycnotic nuclear aggregates result from gaseous pressure exerted within the mitonucleon. It is also assumed that pycnotic nuclei are a sign that a "cell" is dying. While true that the mitonucleon eventually "falls apart", all of the evidence suggests that the DNA is recycled into dome cells so that differentiation, and not apoptosis, explains this example of pycnotic chromatin. 


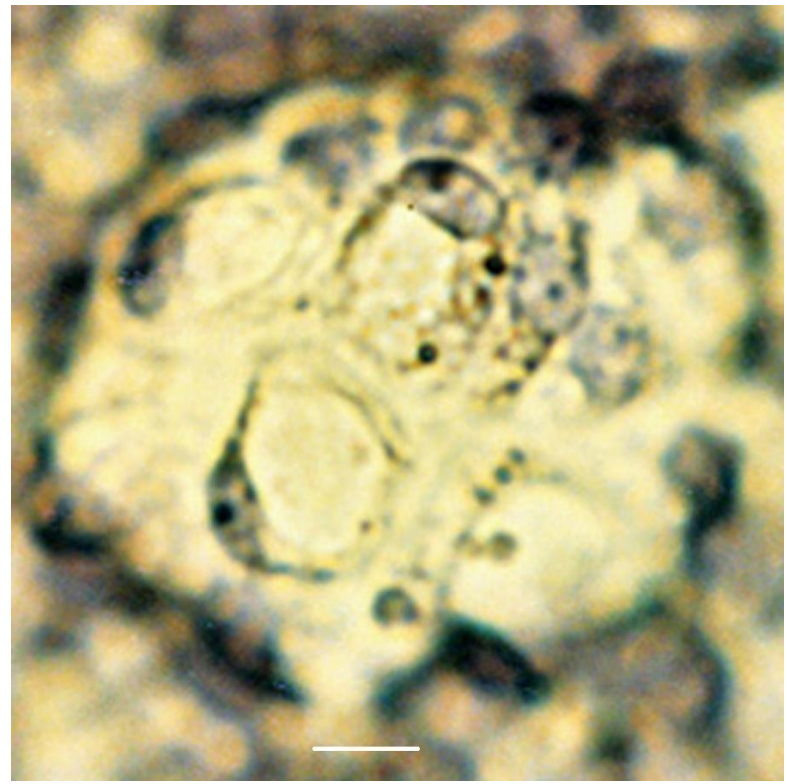

Figure 8. This is what projections such as what is seen in Figure 7 look like when fixed and stained. The profile of the pressured nuclear aggregate can be seen, "flattened" against the the mitonucleon limiting membrane. Gas within the vacuole must be exerting pressure against the nuclear aggregates in the mitonucleons seen at one and seven o'clock in this predome. (Bar $=25$ microns).

Figure 9 is a fixed and stained predome illustrating the final stages in the life of mitonucleons. The mitonucleon on the right is still "completely self-contained," within a double membrane although the disc-shaped nuclear aggregate may have begun to come apart. By contrast, the mitonucleon membrane on the left has been breached. Small dark structures are leaking out into surrounding cytoplasm of what was the syncytium. The heterochromatin has begun to fade in terms of hematoxylin-eosin staining.

Like signet-ring structures, "fading" chromatin has been observed in other cell populations. One of the terms used to describe this intriguing phenomenon is karyolysis, literally the fading of nuclei as observable through H\&E staining. It must be assumed to signal a change in chromatin chemistry. In many systems it is taken as a sign that the cell is dying. In our system, it is associated with DNA fragmentation [17], an essential stage in dome differentiation.

Figure 10 shows a predome with at least 5 mitonucleon protrusions whose structures are coming apart. Chromatin appears to be "fading," losing the ability to take up the hematoxylin or has disappeared completely. Eventually the DNA contained in the mitonucleons is reconstituted into nuclei for the dome cells [18]. Meanwhile during the syncytial elevation, fluid is transported under the basal membrane creating a sustained dome elevation, possibly by an exchange of $\mathrm{H}_{2} \mathrm{O}$ for accumulating gases such as $\mathrm{CO}_{2}[19]$.

The predome in Figure 11 provides another viewpoint on mitonucleon profiles in living culture, neither fixed nor stained. Multiple stages of mitonucleon 


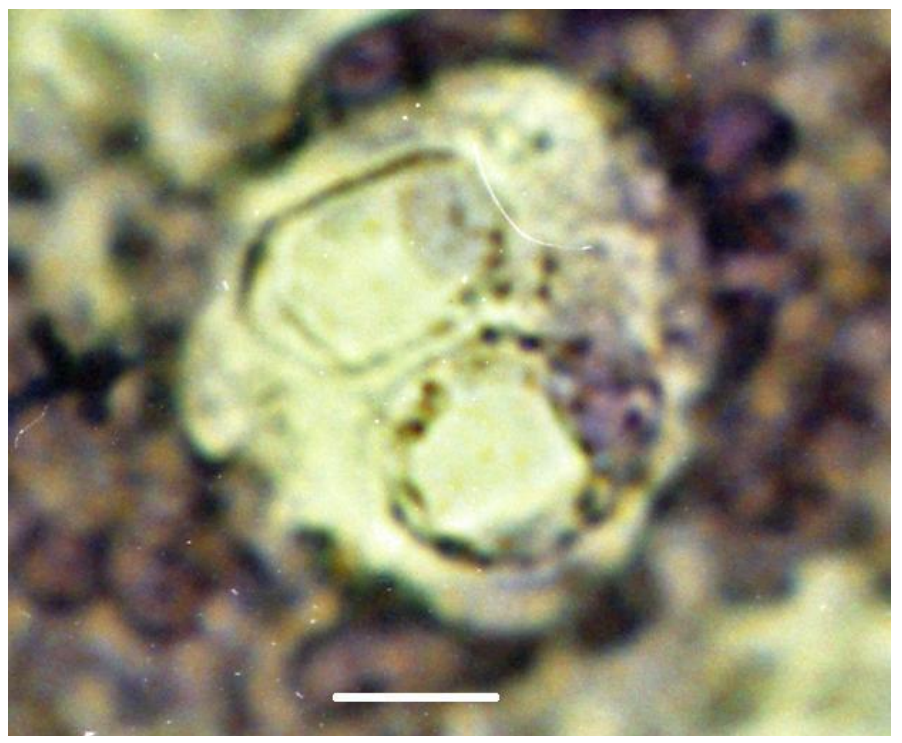

Figure 9. In this fixed and stained predome, close inspection of the nucleon on the right suggests that the previously aggregated nuclei may be coming apart. Nevertheless the mitochondrial superstructure is intact with a central vacuole pressing contents, including the dark structures that look like centrioles against the side of the mitonucleon. In the structure on the left, the mitonucleon wall no longer appears to be a double membrane and the ability of chromatin to take up hematoxylin is fading. (Bar $=25$ microns).

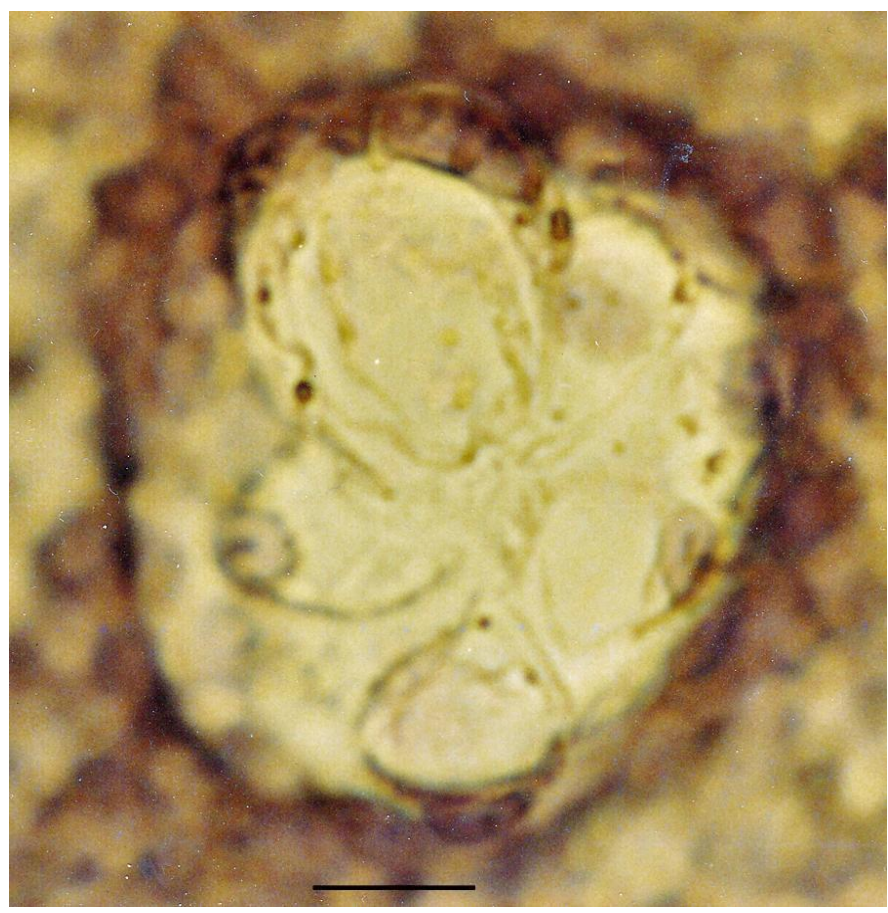

Figure 10. Mitonucleon boundaries are fading as are typical ovoid nuclei. It is at this point, approximately half way through the process of dome formation that the chromatin is broken down into filaments [17] that will be arrayed in the predome and ultimately reassembled into the dome nuclei [18]. (Bar $=25$ microns). 


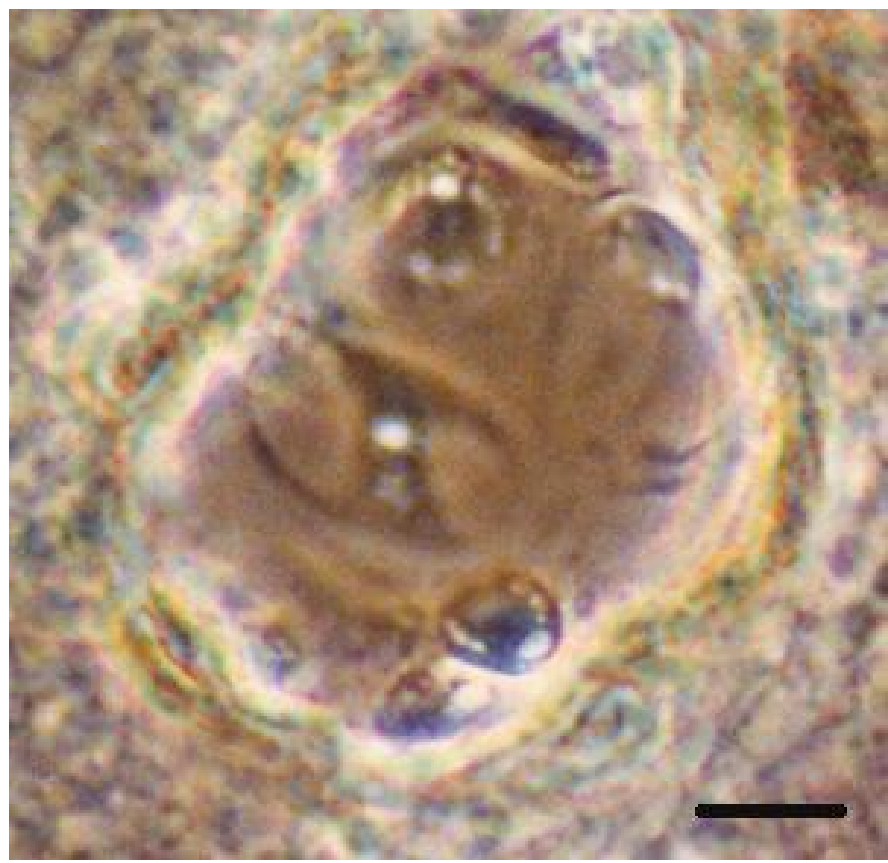

Figure 11. The mitonucleons at twelve o'clock and five o'clock are in the early stages of elevation in a predome. Both show that gas vacuoles form within the chromatin. The mitonucleon at 5 o'clock has a gas vacuole in the center of the chromatin and additional vacuoles. The apical membrane at 1 o'clock is being pushed up by a vacuole, leading with chromatin. The mitonucleon in the center has fallen apart, and its DNA is spreading. Eventually the DNA will be recycled into the cells that make up the dome [18]. (Bar $=25 \mathrm{mi}-$ crons).

development can be observed in this predome. The mitonucleon at 12 o'clock contains vacuoles in the chromatin, but does not appear to have elevated as much as the mitonucleon at 5 o'clock. Similarly vacuolated chromatin has been detected in many different types of epithelial cancers resulting in "cells" that are said to have "optically clear nuclei". So prevalent are they in thyroid cancer that it has been suggested that optically clear nuclei might be a useful marker [20] for thyroid cancer. Given our observation that a mitochondrial membrane wraps around the chromatin, it is possible that the reoriented mitochondrial membrane generates $\mathrm{CO}_{2}$ or other gases that create these unusual vacuoles in the chromatin. Additionally such a mechanism explains how it is that optically clear nuclei are believed to "contain" biotin [21]. The vacuole within chromatin does not appear to be contiguous with the large vacuole pushing up on both the nuclear material and the apical membrane, although each may contain similar mixtures of gas.

The mitonucleon at 1 o'clock appears to have been in the process of elevating, with nuclear material at the top of the projection. Finally, the remains of a mitonucleon can be detected in the center of the predome. The tight ovoid nucleon at the center of the structure has become dispersed. A vacuole can be seen in the center of the mass of chromatin, which is no longer in the projection, instead 
amorphous dense material assumed to be DNA appears to be spreading around membrane projections.

Not shown in this paper, fragmented DNA, previously aggregated, associates with an array of microtubules, filling the large central clearing of the predome [16]. Cell death does not occur. Instead, the fragmented DNA subsequently coalesces into an irregular mass within the envelope of apical and basal membranes under which fluid has been accumulating. From such a chromatin sheet, nuclei emerge by a process that does not appear to involve mitosis.

\section{Discussion}

As shown in this paper, the mitonucleon, a mitochondrial superstructure [5] [13]. forms in response to a stimulus eliciting differentiation in endometrial epithelial cells. The superstructure is responsible for exerting upward pressure on syncytial apical membranes as well as on nuclear aggregates (Figure 4). As the syncytium lifts off the dish (Figure 6) the superstructure rounds up into a spheroid, with fused mitochondria enveloping chromatin. The aggregated chromatin can be seen when a differentiating culture is fixed and stained only with hematoxylin and eosin (Figure 5). Nuclear aggregates in predomes shown in Figure 5 are surrounded by vacuoles that appear to be empty. But the nuclear material takes on a stellate appearance suggesting that it was under pressure from surrounding vacuoles. The speed with which vacuoles arise and the appearance that they are exerting pressure on the nuclear aggregate is consistent with the hypothesis that the vacuoles contain gas [13].

The build-up of gas results in larger vacuoles surrounding chromatin until the entire center of the mitonucleon appears to be a large gas vacuole (Figures 7-9) with smaller gas vacuoles observed in the chromatin (Figure 11). The pressure of gas results in unfurling syncytial membranes (Figure 7) as fluid accumulates under the dome. In the process, cells whose propagation was anchorage-dependent (i.e. growing as a monolayer) will be replaced by cells whose propagation is anchorage-independent (enlarging domes and gland-like structures emerging from the top of the dome).

That these results with cultured endometrial cells are relevant to endometrium in vivo is supported by research done in the middle of the last century. Giant mitochondria were observed in glandular epithelium at the time of ovulation. Armstrong and colleagues established that these giant mitochondria are transient and coincidental with the initiation of the ovarian secretion of progesterone. In cross sections of glandular epithelium, 3 to 4 of these enlarged mitochondria were frequently observed as elongated forms alongside nuclei [8]. Using electron microscopy, researchers also established a relationship between mitochondrial superstructures and endoplasmic reticulum, demonstrating that a single cistern of endoplasmic reticulum (ER) surrounds the mitochondrial superstructures. The single cistern was uniquely semi-rough being studded with ribosomal granules on the membrane facing into the mitochondria and having a 
smooth outer face. Furthermore these researchers showed that the ER connects with surrounding ER and with perinuclear space, establishing a connection between mitochondrial superstructures and extensive membrane systems.

In a subsequent paper from the same laboratory, Coaker et al. demonstrated that the apparently individual giant mitochondria assumed to form by fusion of smaller mitochondria, were also actually connected to each other by sinuous tubular mitochondria [22]. The paper contains models for a complex three dimensional mitochondrial conformation with much twisting, coiling, and budding. The function of mitochondrial superstructures in vivo would appear to be similar to what we report in vitro since they are observed at the beginning of what is called the secretory phase of the menstrual cycle. Our results suggest that they are responsible for gland formation.

\subsection{Are Mitochondrial Superstructures Responsible for Structural Changes Elsewhere?}

The mitochondrial superstructure called nebenkern is involved in morphogenesis of sperm in many insects [3]. It is useful in discussions of mitonucleon function to compare this structure to nebenkern since membrane elongation accompanying sperm tail elaboration may also be dependent on coalescing gas vacuoles. As with mitonucleons, nebenkern were similarly shown to evolve from thread-like mitochondrial structures and giant spheroids, all of which eventually shrink into a compact spherical body. Furthermore the nebenkern literature is full of references to vacuolization and fusing vacuoles. In his 1922 review [1] Bowen cites prior studies demonstrating that vesicular mitochondria fuse together in a darkly staining mass containing many large, non-staining vacuoles.

Mid-century, using improved light microscopes as well as electron microscopy,Tahmisian and colleagues [14] presented evidence that "mitochondria become filamentous and entwine to form the early nebenkern", during grasshopper spermatogenesis. In plate 103 of their paper, the authors show a filamentous mitochondria lying next to nuclear material, an image that looks like Figure 4 in this paper. In plate 107, the authors show vacuoles surrounding the nuclear material. Over time, 8 vacuoles merge into 4 larger vacuoles and then into two very large vacuoles. As described by the authors, "dense nebenkern become less dense .... and numerous large, almost empty, pockets still surrounded by double membranes form. The double membranes separating individual sacs also disappear until only two sacs remain" (whose apparent volume in cross section approximates that of the initial 8 sacs, although the volume clearly increases along the length). The progress of these "sacs" downward may be a motivating force in sperm tail elongation which can, in different insects, amount to more than a thousand-fold increase in length from micron values to centimeter values. Drosophila spermatids start out as a 12 micron wide spherical structure and end up as an 1800 micron long mitochondrial derivative with a greatly enlarged membranous surface area [23]. Significant progress has been made regarding genetic and molecular mechanisms shaping the internal structure of drosophila nebenkern [24]. 
Focusing simply on the membranous surface area the data suggests a simple but elegant mechanism whereby mitonucleon and nebenkern "harness" the power of cell-generated gases to bring about structural changes in cells. Such a mechanism does not call into question the long standing assumption that $\mathrm{CO}_{2}$, a major by-product of metabolism, is usually able to pass freely in and out of cell membranes. But "usually" is not always. Researchers have shown that the variability of membrane composition can result in epithelial apical and basal membranes that differ with regard to $\mathrm{CO}_{2}$ permeability [25]. Is it possible that the surprisingly large and elaborate structures of mitonucleons (perhaps surrounded by endoplasmic reticulum) and nebenkern result in some accumulation of gases for the transient period of their existence?

Our data suggests that the rate of passage of gases out of the superstructure is slowed to the point where gas accumulates within the mitochondrial matrix with $\mathrm{CO}_{2}$ as an obvious candidate. It is understood other gases generated in cells such as $\mathrm{CO}, \mathrm{H}_{2} \mathrm{~S}$, NO that have been shown to play a role in cell physiology. What might be the effect if any of these gases also accumulates? In fact, with all of the fascinating data being accumulated about anaerobic energy metabolism in eukaryotes [26] might $\mathrm{H}_{2}$ itself be generated within these specialized mitochondria if the superstructures provide a transiently anaerobic environment. There is evidence that mitochondria and hydrogenosomes are two forms of the same fundamental organelle [27]. A little more than a decade ago, an anaerobic mitochondrion was shown to produce hydrogen [28]. If a mitochondrial superstructure in an obviously aerobic organism resulted in a brief period when $\mathrm{O}_{2}$ is not passing freely through membranes for the mitochondria making up that structure, might they revert to anaerobic metabolism and the generation of $\mathrm{H}_{2}$. Admittedly speculative, the generation and retention of $\mathrm{H}_{2}$ would certainly enhance buoyancy when generated into membranous structures.

\subsection{Structure of Mitochondrial Matrix that Might Give Rise to Gas Vacuoles}

The dynamic quality of mitochondria [29] has been a topic of interest over the past two decades. The dynamism is not restricted to their ability to fuse into superstructures. Evidence has also accumulated for the existence of at least two different kinds of mitochondrial matrices. Using electron microscopic tomography Frey and Manella [30] [31] showed that mitochondrial cristae can vary from simple tubular structures to more complex lamellar structures with evidence that different structures support different functions. It has been shown that when the inner matrix is tubular, membrane limited subcompartments can form. Our data suggest that the inner matrix of mitonucleons is tubular. More recently data was presented suggesting the existence of different pathways of mitochondrial cristae formation [32].

\subsection{Possible Functions of Other Mitochondrial Superstructures}

Mitochondrial superstructures may serve other functions. There have been, for 
instance, multiple sightings of mitochondrial superstructures in tissues that specialize in the synthesis and excretion of hormones such as the steroids. De Robertis and Sabatini [33] declared that the mitochondrial superstructures found in rat adrenal cortex appeared to result from concentric apposition of flattened laminated mitochondria into structures they called chondriospheres. Furthermore, and relevant to our discussion in this paper, the authors describe large clear vacuoles in these structures. Kadioglu and Harrison [34] explored mitochondrial superstructures in rat adrenal cortex. They discovered vacuolated mitochondrial superstructures that seemed to maintain a steady state of vacuolation. These structures may not be involved in morphogenesis but could be essential when a cell enters into a period of intense anabolic activity. Might gas vacuoles serve as a source for reactants.

Additionally, mitochondrial superstructures have been observed in cells that are being stressed. One theory to explain this finding is that the structures form in response to stress-related increases in free radicals [35].

Finally, structural changes in mitonucleons result in images that are familiar to researchers who study apoptosis [36]. In a preprint, we noted ways in which differentiating Ishikawa cells resemble apoptotic cells [13]. In this regard, it is important to note that caspases, active in apoptosis have been shown to be involved in differentiations that do not lead to cell death [37] [38]. Pyknosis, karyorrhexis and karyolysis, harbingers of programmed cell death in many systems, appear to be driving forces that transform Ishikawa monolayer epithelial cells into differentiated dome cells.

Of the nebenkern, Bowen in his 1922 review [1], lamented that after "thirty five years of observations these appearances are not yet understood." It would appear that there is still much to learn about mitochondrial superstructures after almost 135 years. Since observing mitonucleons in Ishikawa epithelia differentiating into domes, we have detected similar structures generated during spheroid formation, and are currently investigating that phenomenon.

\section{Materials and Methods}

All of the photomicrographs in this paper are of cultured endometrial epithelia called Ishikawa developed by Nishida and colleagues in 1985. His laboratory established that the line contained receptors for both of the female sex steroids, estradiol and progesterone. The cells were obtained from Erlio Gurpide's laboratory at Mt. Sinai, New York. As described in the first paper on the topic of dome formation in 1995 (Fleming), the cells were cultured in phenol red-free Minimum Essential Medium (MEM) supplemented with $2 \mathrm{mM}$ glutamine, $100 \mathrm{U} / \mathrm{ml}$ penicillin, $0.1 \mathrm{mg} / \mathrm{ml}$ and $0.25 \mathrm{mg}$ amphotericin B (GIBCO, Grand Island, NY).

Assays for dome formation were done in confluent cultures, although differentiation has been observed to occur in non confluent cultures. Cells seeded at an approximate density of $5 \times 10^{5} \mathrm{cells} / \mathrm{cm}^{2}$ in MEM containing $5 \%$ calf serum (CS) at 37 degrees $\mathrm{C}$ and $5 \% \mathrm{CO}_{2}$ were grown to confluence. Fetal bovine serum 
(FBS) was more effective in inducing monolayer cells to differentiate than calf serum. FBS was added to confluent monolayers that had rested in $1 \%$ calf serum for 2 to 3 days after reaching confluence. Structures were viewed using an Olympus inverted stage microscope at powers of $100 \times$ (Figure 1(a)), 200× (Figure 1(b)) and 400× (all other figures).

The structures shown in Figure 1(b), Figure 7, and Figure 11 were photographed in living cultures.

Endogenous biotin in the structures shown in Figure 1(a), and Figures 1-4 was detected by an assay using extra-avidin conjugated horseradish peroxidase. Dishes of cells were fixed by adding $4 \%$ paraformaldehyde in phosphate buffered saline (PBS) to culture dishes at various times after they had been stimulated to form domes. After $10 \mathrm{~min}$, the cells were washed gently four times with $5-10 \mathrm{ml}$ PBS. A solution of $1 \%$ Triton X-100 was added to the cells to permeabilize the membrane. Again after $5 \mathrm{~min}$, the culture was washed with successive changes of PBS. After washing, cells were exposed to a 1:200 dilution of Extravidin-conjugated horseradish peroxidase (HRP) (Sigma) for $30 \mathrm{~min}$. After further washing with PBS, a solution of 3-amino-9-ethylcarbazole (20 mg of AEC in 2.5 $\mathrm{ml}$ of dimethylformamide, diluted with $47.5 \mathrm{ml}$ of $50 \mathrm{mM}$ potassium acetate adjusted to $\mathrm{pH} 5.0$ ) was added to the cells together with $0.25 \% \mathrm{H}_{2} \mathrm{O}_{2}$. This solution was incubated at $37 \mathrm{C}$ for $45 \mathrm{~min}$ to allow color to develop. The AEC solution was removed, and the cultures were examined and stored in the presence of PBS at 4 "C. Additionally, cells were stained with a solution of hematoxylin and eosin.

If avidin linked to peroxidase is not added to the cultures, there is no reaction. If avidin without peroxidase is added first to the cultures, followed by avidin-linked to peroxidase, staining is not observed. Staining does not occur if avidin-HRP is not added to the cultures prior to AEC indicating that an endogenous peroxidase is not responsible for the staining. To ensure that avidin was reacting with biotin, we stained domes using streptavidin linked to horseradish peroxidase as well as primary antibody to biotin and secondary antibody-linked to horseradish peroxidase. Staining occurred under all circumstances, indicating that avidin does indeed react with biotin that is endogenously present in cells in significant amounts.

The cells in Figure 5, Figures 8-10 were fixed as described and stained only with hematoxylin and eosin.

\section{References}

[1] Bowen R.H. (1922) Studies on Insect Spermatogenesis III. On the Structure of the Nebenkern in the Insect Spermatid and the Origin of Nebenkern Patterns. Biological Bulletin, 42, 53-84.

[2] Fabian, L. and Brill, J.A. (2012) Drosophila Spermiogenesis: Big Things Come from Little Packages. Spermatogenesis, 2, 197-212. https://doi.org/10.4161/spmg.21798

[3] Phillips, D.M. (1970) Insect Sperm: Their Structure and Morphogenesis. The Journal of Cell Biology, 44, 243-277. https://doi.org/10.1083/jcb.44.2.243

[4] Nishida, M., Kasahara, K., Kaneko, M. and Iwasaki, H. (1985) Establishment of a 
New Human Endometrial Adenocarcinoma Cell Line, Ishikawa Cells, Containing Estrogen and Progesterone Receptors. Acta Obstetrica et Gynaecologica Japonica, 37, 1103.

[5] Fleming, H., Condon, R., Peterson, G., Guck, I., Prescott, E., Chatfield, K. and Duff, M. (1998) Role of Biotin-Containing Membranes and Nuclear Distribution in Differentiating Human Endometrial Cells. Journal of Cellular Biochemistry, 71, 400-415.

https://doi.org/10.1002/(SICI)1097-4644(19981201)71:3\%3C400::AID-JCB9\%3E3.0. $\mathrm{CO} ; 2-\mathrm{W}$

[6] Fleming, H. (1995) Differentiation in Human Endometrial Cells in Monolayer Culture: Dependence on a Factor in Fetal Bovine Serum. Journal of Cellular Biochemistry, 57, 262-270. https://doi.org/10.1002/jcb.240570210

[7] Fleming, H. (1999) Structure and Function of Cultured Endometrial Epithelial Cells. Seminars in Reproductive Endocrinology, 17, 93-106.

https://doi.org/10.1055/s-2007-1016215

[8] Armstrong, E.M., More, I.A., McSeveney, D. and Carty, M. (1973) The Giant Mitochondrion-Endoplasmic Reticulum Unit of the Human Endometrial Glandular Cell. Journal of Anatomy, 116, 375-383.

[9] Neuspiel, M., Schauss, A.C., Braschi, E., Zunino, R., Rippstein, P., Rachubinski, R.A., Andrade-Navarro, M.A. and McBride, H.M. (2008) Cargo-Selected Transport from the Mitochondrial Peroxisomes Is Mediated by Vesicular Carriers. Current Biology, 18, 102-108.

[10] Andrade-Navarro, M.A., Sanchez-Pulido, L. and McBride, H.M. (2009) Mitochondrial Vesicles: An Ancient Process Providing New Links to Peroxisomes. Current Opinion in Cell Biology, 4, 560-567.

[11] Sugiura, A., McLelland, G.L., Fon, E.A. and McBride, H.M. (2014) A New Pathway for Mitochondrial Quality Control: Mitochondrial-Derived Vesicles. The EMBO Journal, 33, 2142-2156.

[12] Hailey, D.W., Kim, P.K., Satpute-Krishnan, P., Rambold, A.S., Mitra, K., Sougrat, R. and Lippincott-Schwartz, J. (2010) Mitochondria Supply Membranes for Autophagosome Biogenesis during Starvation. Cell, 141, 656-667. https://doi.org/10.1016/j.cell.2010.04.009

[13] Fleming, H. (2016) Mitonucleons Formed during Differentiation of Ishikawa Endometrial Cells Generate Vacuoles that Elevate Monolayer Syncytia: Differentiation of Ishikawa Domes, Part 1. PeerJ Preprints, 4, e1728v1.

[14] Tahmisian, T.L., Powers, E.L. and Devine, R.L. (1956) Light and Electron Microscope Studies of MOrphological Changes of Mitochondria during Spermatogenesis in the Grasshopper. The Journal of Biophysical and Biochemical Cytology, 2, 325-350. https://doi.org/10.1083/jcb.2.4.325

[15] Kedzior, J., Masaoka, M., Kurono, C., Spodnik, J.H., Hallmann, A., Majczak, A., Niemczyk, E., Trzonkowski, P., Myśliwski, A., Soji, T. and Wakabayashi, T. (2004) Changes in Physicochemical Properties of Microtubules Lead to the Formation of a Single Spherical Structure of Mitochondrial Assembly Enveloping Nuclear Chromatins. Journal of Electron Microscopy ( Tokyo), 53, 659-670.

https://doi.org/10.1093/jmicro/dfh087

[16] Grilliot, M.A., Goldblum, J.R. and Liu, X. (2012) Signet-Ring Cell Melanoma of the Gastroesophageal Junction: A Case Report and Literature Review. Archives of Pathology \& Laboratory Medicine, 136, 324-328. https://doi.org/10.5858/arpa.2011-0042-CR 
[17] Fleming, H. (2016) Pyknotic Chromatin in Mitonucleons Elevating in Syncytia Undergo Karyorhhexis and Karyolysis before Coalescing into an Irregular Chromatin Mass: Differentiation of Ishikawa Domes, Part 2. PeerJ Preprints, 4, e1729v1.

[18] Fleming, H. (2016) Chomatin Mass from Previously Aggregated, Pyknotic, and Fragmented Monolayer Nuclei Is a Source for Dome Cell Nuclei Generated by Amitosis: Differentiation of Ishikawa Domes, Part 3. PeerJ Preprints, 4, e1730v1.

[19] Chen, L.-M., Zhao, J., Musa-Aziz, R., Pelletier, M.F., Drummond, I.A. and Boron, W.F. (2010) Cloning and Characterization of a Zebrafish Homologue of Human AQP1: A Bifunctional Water and Gas Channel. American Journal of Physiology-Regulatory, Integrative and Comparative Physiology, 299, R1163-R1174. https://doi.org/10.1152/ajpregu.00319.2010

[20] Hapke, M.R. and Dehner, L.P. (1979) The Optically Clear Nucleus: A Reliable Sign of Papillary Carcinoma of the Thyroid? The American Journal of Surgical Pathology, 1, 31-38. https://doi.org/10.1097/00000478-197902000-00004

[21] Nakatani, Y., Kitamura, H., Inayama, Y. and Ogawa, N. (1994) Pulmonary Endodermal Tumor Resembling Fetal Lung. The Optically Clear Nucleus Is Rich in Biotin. The American Journal of Surgical Pathology, 18, 637. https://doi.org/10.1097/00000478-199406000-00008

[22] Coaker, T., Downie, I. and More, A.R. (1982) Complex Giant Mitochondria in the Human Endometrial Glandular Cell: Serial Sectioning, High-Voltage Electron Microscopic, and Three-Dimensional Reconstruction Studies. Journal of Ultrastructure Research, 78, 283-291. https://doi.org/10.1016/S0022-5320(82)80003-8

[23] Noguchi, T., Koizumi, M. and Hayashi, S. (2011) Sustained Elongation of Sperm Tail Promoted by Local Remodeling of Giant Mitochondria in Drosophila. Current Biology, 21, 805-814. https://doi.org/10.1016/j.cub.2011.04.016

[24] Sawyer, E.M., Brunner, E.C., Hwang, Y., Ivey, L.E., Brown, O., Bannon, M., Akrobetu, D., Sheaffer, K.E., Morgan, O., Field, C.O., Suresh, N., Gordon, G., Gunnell, E.T., Regruto, L.A., Wood, C.A., Fuller, M.T. and Hales, K.G. (2017) Testis-Specific ATP Synthase Peripheral Stalk Subunits Required for Tissue-Specific Mitochondrial Morphogenesis in Drosophila. BMC Cell Biology, 18, 16. https://doi.org/10.1186/s12860-017-0132-1

[25] Endeward, V. and Gros, G. (2013) Low Carbon Dioxide Permeability of the Apical Epithelial Membrane of Guinea Pig Colon. Frontiers in Physiology, 4, 382. https://doi.org/10.3389/fphys.2013.00382

[26] Müller, M., Mentel, M., van Hellemond, J.J., Henze, K., Woehle, C., Gould, S.B., Re-Young, Y., van der Giezen, M., Tielens, A.G.M. and Martin, W.F. (2012) Biochemistry and Evolution of Anaerobic Energy Metabolism in Eukaryotes. Microbiology and Molecular Biology Reviews. MMBR, 76, 444-495. https://doi.org/10.1128/MMBR.05024-11

[27] Embley, T.M., van der Giezen, M., Horner, D.S., Dyal, P.L. and Foster, P. (2003) Mitochondria and Hydrogenosomes Are Two Forms of the Same Fundamental Organelle. Philosophical Transactions of the Royal Society B: Biological Sciences, 358, 191-202. https://doi.org/10.1098/rstb.2002.1190

[28] Boxma, B., de Graaf, R.M., van der Staay, G.W., van Alen, T.A., Ricard, G., Gabaldón, T., van Hoek, A.H., Moon-van der Staay, S.Y., Koopman, W.J., van Hellemond, J.J., Tielens, A.G., Friedrich, T., Veenhuis, M., Huynen, M.A. and Hackstein, J.H. (2005) An Anaerobic Mitochondrion That Produces Hydrogen. Nature, 434, 74-79.

[29] Bereiter-Hahn, J. and Vöth, M. (1994) Dynamics of Mitochondria in Living Cells: 
Shape Changes, Dislocations, Fusion, and Fission of Mitochondria. Microscopy Research and Technique, 27, 198-219. https://doi.org/10.1002/jemt.1070270303

[30] Frey, T.G. and Mannella, C.A. (2000) The Internal Structure of Mitochondria. Trends in Biochemical Sciences, 25, 319-324. https://doi.org/10.1016/S0968-0004(00)01609-1

[31] Mannella, C.A. (2008) Structural Diversity of Mitochondria: Functional Implications. Annals of the New York Academy of Sciences, 1147, 171-179. https://doi.org/10.1196/annals.1427.020

[32] Harner, M.E., Unger, A.-K., Geerts, W.J., Mari, M., Izawa, T., Stenger, M., Neupert, W., et al. (2016) An Evidence Based Hypothesis on the Existence of Two Pathways of Mitochondrial Crista Formation. eLife, 5, e18853. https://doi.org/10.7554/eLife.18853

[33] De Robertis, E. and Sabatini, D. (1958) Mitochondrial Changes in the Adrenocortex of Normal Hamsters. The Journal of Biophysical and Biochemical Cytology, 4, 667-670. https://doi.org/10.1083/jcb.4.5.667

[34] Kadioglu, D. and Harrison, R.G. (1971) The Functional Relationships of Mitochondria in the Rat Adrenal Cortex. Journal of Anatomy, 110, 283-296.

[35] Wakabayashi, T. (2002) Megamitochondria Formation-Physiology and Pathology. Journal of Cellular and Molecular Medicine, 6, 497-538. https://doi.org/10.1111/j.1582-4934.2002.tb00452.x

[36] Sun, M.G., Williams, J., Munoz-Pinedo, C., Perkins, G.A., Brown, J.M., Ellisman, M.H., Green, D.R. and Frey, T. (2007) Correlated Three-Dimensional Light and Electron Microscopy Reveals Transformation of Mitochondria during Apoptosis. Nature Cell Biology, 9, 1057-1065. https://doi.org/10.1038/ncb1630

[37] Fernando, P. and Megeney, L.A. (2006) Is Caspase Dependent Apoptosis Only Cell Differentiation Taken to the Extreme? The FASEB Journal, 21, 8-17. https://doi.org/10.1096/fj.06-5912hyp

[38] Murray, T.V., McMahon, J.M., Howley, B.A., Stanley, A., Ritter, T., Mohr, A., Zwacka, R. and Fearnhead, H.O. (2008) A Non-Apoptotic Role for Caspase-9 in Muscle Differentiation. Journal of Cell Science, 121, 3786-3793. https://doi.org/10.1242/jcs.024547 